\title{
ON COLLISION-AVOIDING INITIAL CONFIGURATIONS TO CUCKER-SMALE TYPE FLOCKING MODELS*
}

\author{
SHIN MI AHN ${ }^{\dagger}$, HEESUN CHOI ${ }^{\ddagger}$, SEUNG-YEAL HA ${ }^{\S}$, AND HO LEE
}

\begin{abstract}
We present a class of initial-configurations for the Cucker-Smale flocking type models leading no finite-time collisions between particles. For this class of initial-configurations, the global existence of smooth solutions to the Cucker-Smale model with singular communication weights is established. This also generalizes the earlier flocking estimates for the Cucker-Smale type models with regular communication weights.
\end{abstract}

Key words. The Cucker-Smale model, inter-particle bonding force, collision-avoidance, configuration energy.

AMS subject classifications. 92D25, 74A25, 76N10.

\section{Introduction}

Flocking phenomena $[3,11,12,13,14,32,33,34]$ such as flocking of birds, schooling of fishes, and swarming of bacteria are often observed in complex biological systems, and are some of the collective self-organizing phenomena in biological, mechanical, and social groups of self-propelled particles. Recently several mathematical models for such self-organized phenomena have received lots of attention due to their engineering applications such as sensor networks, and formation control of robot and unmanned aerial vehicles $[26,28,30]$. Our interest in this paper lies on the flocking model introduced by Cucker and Smale [10]. They proposed a simple continuoustime dynamical system (called C-S model) with regular and algebraically decaying communication weight depending on the Euclidean distance between agents and provide rigorous asymptotic flocking estimates exhibiting a kind of phase-transition like phenomena between local flocking and global flocking depending on the decay rate of communication weight (e.g. long range and short range). Cucker-Smale's flocking estimates were refined and generalized to the kinetic and fluid models in recent literature $[1,2,4,5,6,7,8,9,15,16,17,18,19,20,21,22,24,25,27,31]$. In application of the C-S model for the UAV and robot system, there are several difficulties. Among them, collision avoidance and existence of too many spatial equilibria can be mentioned. For collision avoidance in the C-S model, Cucker and Dong recently introduced a modified C-S model by adding a term which prevent collisions [7], and for the second issue Park-Kim-Ha also introduced a C-S model with inter-particle bonding forces [29]. When the communication weight is adopted as a singular type, say $\frac{1}{r^{\alpha}}, \alpha>0$, the forcing terms become singular in the original C-S model and its variants [7, 29]. Hence control of pair-wise collisions is necessary for the well-posedness of the models. Then natural questions which arise are (1) what is the effect of singularity of communication weights?, (2) can it prevent collisions? or (3) even for singular weights, if

${ }^{*}$ Received: April 3, 2011; accepted (in revised version): August 16, 2011. Communicated by Pierre Degond.

${ }^{\dagger}$ Department of Mathematical Sciences, Seoul National University, Seoul 151-747, Korea (ahn1004@math.snu.ac.kr).

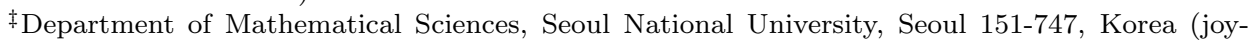
ful25@snu.ac.kr).

$\S$ Department of Mathematical Sciences, Seoul National University, Seoul 151-747, Korea (syha@snu.ac.kr).

『Max-Planck-Institute for Gravitational Physics, Albert-Einstein-Institute, Am Mühlenberg 1, D-14476 Golm, Germany (ho.lee@aei.mpg.de). 
collisions can happen, then can we identify admissible initial configurations to prevent collision in finite-time? etc.

The main purpose of this paper is to characterize an admissible class of initial configurations preventing finite-time collisions. For the simple two-particle C-S system, the non-integrable singularity of communication weights can prevent a finite-time collision for the configurations once the two particles are initially apart (Proposition 4.1).

The rest of this paper is divided into five sections after the introduction. In Section 2, we introduce a C-S type flocking model with non-integrable singular communication weights and inter-particle bonding forces and discuss a framework for collision avoidance. In Section 3, we present $\ell_{\infty}$-flocking estimates for the original C-S model with regular communication weights, which improves the results in [5, 22]. In Section 4, we introduce an admissible class of initial-configurations for the collision avoidance for the C-S model with singular communication weights. For many-body particle systems, we present a sufficient condition for collision avoidance by providing the uniform positive lower bound for the minimal distance between particles thanks to the explicit exponential time-decay of the relative velocities. In Section 5, we establish that the maximal inter-particle distance can be controlled by the minimal inter-particle distance by devising a new Lyapunov functional. This estimate is reminiscent of "Harnack type estimate for positive harmonic functions" in the elliptic PDE theory. Thanks to a new a priori estimate, we can show that pair-wise collision induces a one-point collapse of the particle system, which is impossible for the C-S model with inter-particle bonding force when the total energy satisfies a bound condition. Finally Section 6 summarizes the main results of this paper. Notations:

Throughout the paper, we use the superscript to denote the component of a vector, for example $\boldsymbol{x}:=\left(x^{1}, \cdots, x^{d}\right) \in \mathbb{R}^{d}$. For vectors $\boldsymbol{x}, \boldsymbol{v} \in \mathbb{R}^{d}$, their Euclidean norm and the inner product are defined as follows:

$$
\|\boldsymbol{x}\|:=\left(\sum_{i=1}^{d}\left(x^{i}\right)^{2}\right)^{\frac{1}{2}}, \quad\langle\boldsymbol{x}, \boldsymbol{v}\rangle:=\sum_{i=1}^{d} x^{i} v^{i},
$$

where $x^{i}$ and $v^{i}$ are the $i$-th components of $\boldsymbol{x}$ and $\boldsymbol{v}$ respectively.

\section{Preliminaries}

In this section, we present two Cucker-Smale (C-S) type flocking models with singular communication weights and bonding forces and discuss structural assumptions on $\psi$.

2.1. The C-S model with a singular weight. In this part, we consider the C-S model equipped with singular communication weights. Consider an interacting particle system consisting of $N$-identical point particles with unit mass in $\mathbb{R}^{d}$, and let $\left(\boldsymbol{x}_{i}(t), \boldsymbol{v}_{i}(t)\right) \in \mathbb{R}^{2 d}$ be the phase-space position of the $i$-th agent. Then the C-S dynamical system reads as follows:

$$
\begin{aligned}
& \frac{d \boldsymbol{x}_{i}}{d t}=\boldsymbol{v}_{i}, \quad t>0, \quad i=1, \cdots, N, \\
& \frac{d \boldsymbol{v}_{i}}{d t}=\frac{K_{0}}{N} \sum_{\substack{j \neq i \\
j=1}}^{N} \psi\left(r_{i j}\right)\left(\boldsymbol{v}_{j}-\boldsymbol{v}_{i}\right),
\end{aligned}
$$


subject to initial data

$$
\left(\boldsymbol{x}_{i}, \boldsymbol{v}_{i}\right)(0)=\left(\boldsymbol{x}_{i 0}, \boldsymbol{v}_{i 0}\right),
$$

where $r_{i j}:=\left\|\boldsymbol{x}_{i}-\boldsymbol{x}_{j}\right\|$ and $K_{0}$ is the positive coupling strength. The function $\psi=\psi(r)$ denotes the communication weight between particles whose relative distance is exactly $r$. Note that when the function $\psi$ is bounded and locally Lipschitz, the standard Cauchy-Lipschitz theory guarantees the existence of global smooth solution to the system (2.1)-(2.2). However, when the weight function is not locally bounded, the existence of global solutions is not clear. For example, for $\psi(r)=\frac{1}{r^{\alpha}}, \alpha>0$, when $r \rightarrow 0$, i.e., two particles are approaching each other, the value of the weight function becomes infinity, hence the standard global existence theory breaks down, so there is no global smooth solution after the collision time. Therefore, for singular weights we need to choose suitable initial configurations which do not collide in finite time to guarantee the global existence of smooth solutions.

2.2. The C-S model with a bonding force. In this part, we briefly discuss the particle model introduced in [29], which is called a C-S flocking model with inter-particle bonding forces. In this model, we have incorporated new additional interaction terms confining particles. The model reads as follows:

$$
\begin{aligned}
\frac{d \boldsymbol{x}_{i}}{d t}= & \boldsymbol{v}_{i}, \quad t>0, \quad i=1, \cdots, N, \\
\frac{d \boldsymbol{v}_{i}}{d t}= & \frac{K_{0}}{N} \sum_{\substack{j \neq i \\
j=1}}^{N} \psi\left(r_{i j}\right)\left(\boldsymbol{v}_{j}-\boldsymbol{v}_{i}\right)+\frac{K_{1}}{N} \sum_{\substack{j \neq i \\
j=1}}^{N} \frac{1}{2 r_{i j}^{2}}\left\langle\boldsymbol{v}_{i}-\boldsymbol{v}_{j}, \boldsymbol{x}_{i}-\boldsymbol{x}_{j}\right\rangle\left(\boldsymbol{x}_{j}-\boldsymbol{x}_{i}\right) \\
& +\frac{K_{2}}{N} \sum_{\substack{j \neq i \\
j=1}}^{N} \frac{1}{2 r_{i j}}\left(r_{i j}-2 R\right)\left(\boldsymbol{x}_{j}-\boldsymbol{x}_{i}\right),
\end{aligned}
$$

where $K_{i}, i=0,1,2$ are positive constants measuring the coupling strength. Here $R$ is a control parameter to make particles stay away from each other. It is easy to see that for a two-particle system, $2 R$ is exactly asymptotic inter-particle distance. Note that the new terms

$$
\frac{K_{1}}{N} \sum_{\substack{j \neq i \\ j=1}}^{N} \frac{1}{2 r_{i j}^{2}}\left\langle\boldsymbol{v}_{i}-\boldsymbol{v}_{j}, \boldsymbol{x}_{i}-\boldsymbol{x}_{j}\right\rangle\left(\boldsymbol{x}_{j}-\boldsymbol{x}_{i}\right)+\frac{K_{2}}{N} \sum_{\substack{j \neq i \\ j=1}}^{N} \frac{1}{2 r_{i j}}\left(r_{i j}-2 R\right)\left(\boldsymbol{x}_{j}-\boldsymbol{x}_{i}\right)
$$

were devised to control spatial configurations for the particle system (formation control) in [29]. These terms force particles to remain about $2 R$ units apart in the low dimensional case. Of course, this may not be true when the number of particles is large. Nevertheless, these terms are expected to control the distances between particles, so that the particles do not collide with each other. In [29], the possibility of finite-collision avoidance was not ruled out, although total collapse can be avoided for some class of initial data. This issue will be treated in Section 5 .

Definition 2.1. [22] A multi-agent system $\mathcal{P}:=\left\{\left(\mathbf{x}_{i}, \boldsymbol{v}_{i}\right)\right\}_{i=1}^{N}$ has an asymptotic flocking if and only if the system $\mathcal{P}$ satisfies the following two conditions:

(i) (Velocity alignment) The velocity diameter of the set $\left\{\boldsymbol{v}_{k}\right\}_{k=1}^{N}$ goes to zero as time goes to infinity:

$$
\lim _{t \rightarrow \infty}\left\|\boldsymbol{v}_{j}(t)-\boldsymbol{v}_{i}(t)\right\|=0
$$


(ii) (Forming a group) The spatial diameter of the set $\left\{\boldsymbol{x}_{k}\right\}_{k=1}^{N}$ is uniformly bounded in time $t$ :

$$
\sup _{0 \leq t<\infty}\left\|\boldsymbol{x}_{i}(t)-\boldsymbol{x}_{j}(t)\right\|<\infty .
$$

Before we close this section, we present an example to exhibit the possibility of finite-time collisions in the Cucker-Smale model.

Example (Occurrence of finite-time collisions). Consider a simple two-particle system with a communication weight $\psi \equiv 1$ :

$$
\begin{array}{ll}
\frac{d x_{1}}{d t}=v_{1}, & \frac{d v_{1}}{d t}=\frac{K}{2}\left(v_{2}-v_{1}\right), \\
\frac{d x_{2}}{d t}=v_{2}, & \frac{d v_{2}}{d t}=\frac{K}{2}\left(v_{1}-v_{2}\right) .
\end{array}
$$

We set

$$
x:=x_{1}-x_{2}, \quad v:=v_{1}-v_{2} .
$$

Then we have

$$
\frac{d x}{d t}=v, \quad \frac{d v}{d t}=-K v .
$$

Suppose the initial data satisfies

$$
x_{0}>0, \quad v_{0}<0, \quad K<\frac{\left|v_{0}\right|}{\left|x_{0}\right|} .
$$

Then by direct calculation, we have an explicit solution:

$$
v(t)=e^{-K t} v_{0}, \quad x(t)=x_{0}+\frac{v_{0}}{K}\left(1-e^{-K t}\right) .
$$

Note that

$$
0=x_{0}+\frac{v_{0}}{K}\left(1-e^{-K t}\right) \quad \Longleftrightarrow \quad t=\frac{1}{K} \ln \left(\frac{\left|v_{0}\right|}{\left|v_{0}\right|-K\left|x_{0}\right|}\right) .
$$

Hence for initial data satisfying (2.5), we have a finite-time collision at $t_{*}=$ $\frac{1}{K} \ln \left(\frac{\left|v_{0}\right|}{\left|v_{0}\right|-K\left|x_{0}\right|}\right)<\infty$.

\section{A generalized flocking estimate for the C-S model}

In this section, we present a generalized flocking estimate for the C-S model with a regular communication weight. Since the total momentum for the C-S model (2.1) is conserved along the flow, we may assume

$$
\sum_{i=1}^{N} \boldsymbol{x}_{i}(t)=0, \quad \sum_{i=1}^{N} \boldsymbol{v}_{i}(t)=0, \quad t \geq 0 .
$$

If necessary, we consider the fluctuations $\boldsymbol{x}_{i}-\boldsymbol{x}_{c}$ and $\boldsymbol{v}_{i}-\boldsymbol{v}_{c}$ satisfying the above constraints instead of working with $\boldsymbol{x}_{i}, \boldsymbol{v}_{i}$. In [22], a Lyapunov functional approach has been introduced in the $\ell_{2}$-framework for regular weights. Hence the estimates do not hold for $N=\infty$, because in this limit the admissible initial configurations 
become trivial. However this difficulty was resolved for the $\ell_{\infty}$-framework in [5] for the Cucker-Smale communication weight:

$$
\psi_{c s}(r):=\left(1+r^{2}\right)^{-\frac{\beta}{2}}, \quad \beta \geq 0 .
$$

In the sequel, we combine the Lyapunov functional approach in [22] together with the $\ell_{\infty}$-approach in [5] to obtain a generalized flocking estimate for general class of communication weights. Throughout this section, we assume that the communication weight $\psi$ is positive, bounded, non-increasing, and Lipschitz continuous:

$$
\psi\left(r_{1}\right) \geq \psi\left(r_{2}\right), \quad r_{1} \leq r_{2}, \quad 0<\psi(r) \leq \psi_{\infty}, \quad r>0 .
$$

3.1. Lyapunov type functionals in the $\ell_{\infty}$-framework. In this part, we define a Lyapunov functional in the $\ell_{\infty}$-framework. The first step, as in [22], is to derive a system of two differential inequalities. For this, we set a mixed norm as follows:

$$
\|\boldsymbol{x}\|_{\infty}:=\max _{1 \leq i \leq N}\left\|\boldsymbol{x}_{i}\right\|, \quad\|\boldsymbol{v}\|_{\infty}:=\max _{1 \leq i \leq N}\left\|\boldsymbol{v}_{i}\right\|,
$$

where $\boldsymbol{x}$ and $\boldsymbol{v}$ are vectors in $\mathbb{R}^{N d}$ :

$$
\boldsymbol{x}:=\left(\boldsymbol{x}_{1}, \cdots, \boldsymbol{x}_{N}\right), \quad \boldsymbol{v}:=\left(\boldsymbol{v}_{1}, \cdots, \boldsymbol{v}_{N}\right) \in \mathbb{R}^{N d} .
$$

Note that the maximal indices are varying depending on $t,\|\boldsymbol{x}\|_{\infty}$ and $\|\boldsymbol{v}\|_{\infty}$ are piecewise smooth functions, and by the analyticity of $\boldsymbol{x}_{i}$ and $\boldsymbol{v}_{i}$, the non-differential points of $\|\boldsymbol{x}\|_{\infty}$ and $\|\boldsymbol{v}\|_{\infty}$ are at most countable and discrete. In the following lemma, we will show that the two scalar functionals $\|\boldsymbol{x}\|_{\infty}$ and $\|\boldsymbol{v}\|_{\infty}$ satisfy a system of two differential inequalities as long as $\left(\boldsymbol{x}_{i}, \boldsymbol{v}_{i}\right)$ is a solution of the system (2.1).

Lemma 3.1. Suppose (3.1) and (3.2) hold, and let $\left\{\left(\mathbf{x}_{i}, \boldsymbol{v}_{i}\right)\right\}_{i=1}^{N}$ be the global smooth solution to the system (2.1). Then $\|\boldsymbol{x}\|_{\infty}$ and $\|\boldsymbol{v}\|_{\infty}$ satisfy

$$
\left|\frac{d\|\boldsymbol{x}\|_{\infty}}{d t}\right| \leq\|\boldsymbol{v}\|_{\infty}, \quad \frac{d\|\boldsymbol{v}\|_{\infty}}{d t} \leq-K_{0} \psi\left(2\|\boldsymbol{x}\|_{\infty}\right)\|\boldsymbol{v}\|_{\infty}, \quad \text { a.e. } t \in[0, \infty) .
$$

Proof.

(i) We set $\left\{t_{i}\right\}$ to be the set of times satisfying

$$
0=t_{0}<t_{1}<\cdots<t_{n}<\cdots, \quad\|\boldsymbol{x}\|_{\infty} \text { is differentiable in }\left(t_{i-1}, t_{i}\right), \quad i=1, \ldots
$$

In the time-interval $\left(t_{i-1}, t_{i}\right)$, we choose $k \in\{1, \ldots, N\}$ to satisfy

$$
\|\boldsymbol{x}(t)\|_{\infty}=\left\|\boldsymbol{x}_{k}(t)\right\|, \quad t \in\left(t_{i-1}, t_{i}\right) .
$$

Then we use the Cauchy-Schwartz inequality to obtain

$$
\begin{aligned}
\left|2\left\|\boldsymbol{x}_{k}\right\| \frac{d\left\|\boldsymbol{x}_{k}\right\|}{d t}\right| & =\left|\frac{d\left\|\boldsymbol{x}_{k}\right\|^{2}}{d t}\right|=2\left|\left\langle\boldsymbol{x}_{k}, \frac{d \boldsymbol{x}_{k}}{d t}\right\rangle\right| \\
& =2\left|\left\langle\boldsymbol{x}_{k}, \boldsymbol{v}_{k}\right\rangle\right| \leq 2|| \boldsymbol{x}_{k}|||| \boldsymbol{v}_{k} \|, \quad t \in\left(t_{i-1}, t_{i}\right) .
\end{aligned}
$$

This yields

$$
\left|\frac{d\left\|\boldsymbol{x}_{k}\right\|}{d t}\right| \leq\left\|\boldsymbol{v}_{k}\right\| \leq\|\boldsymbol{v}\|_{\infty}, \quad t \in\left(t_{i-1}, t_{i}\right)
$$


Hence we obtain the desired inequality.

(ii) For the second inequality, we use a similar argument to (i) and an energy estimate. We set $\left\{s_{i}\right\}$ to be the set of times satisfying

$$
0=s_{0}<s_{1}<\cdots<s_{n}<\cdots, \quad\|\boldsymbol{v}\|_{\infty} \text { is differentiable in }\left(s_{i-1}, s_{i}\right), \quad i=1, \ldots .
$$

In the time-interval $\left(s_{i-1}, s_{i}\right)$, we choose $k \in\{1, \cdots, N\}$ to satisfy

$$
\|\boldsymbol{v}(t)\|_{\infty}=\left\|\boldsymbol{v}_{k}(t)\right\|, \quad t \in\left(s_{i-1}, s_{i}\right) .
$$

Then it follows from the momentum equation in (2.1) that

$$
\begin{aligned}
& \frac{d}{d t}\left\|\boldsymbol{v}_{k}(t)\right\|^{2} \\
= & 2\left\langle\boldsymbol{v}_{k}(t), \frac{d \boldsymbol{v}_{k}(t)}{d t}\right\rangle \\
= & -\frac{2 K_{0}}{N} \sum_{j=1}^{N} \psi\left(\left\|\boldsymbol{x}_{j}(t)-\boldsymbol{x}_{k}(t)\right\|\right)\left\langle\boldsymbol{v}_{k}(t)-\boldsymbol{v}_{j}(t), \boldsymbol{v}_{k}(t)\right\rangle \\
\leq & -2 K_{0} \psi\left(2\|\boldsymbol{x}(t)\|_{\infty}\right)\|\boldsymbol{v}(t)\|_{\infty}^{2},
\end{aligned}
$$

where we used (3.1) and the choice of $\boldsymbol{v}_{k}$ to see

$$
\begin{aligned}
\psi\left(\left\|\boldsymbol{x}_{j}-\boldsymbol{x}_{k}\right\|\right) & \geq \psi\left(2\|\boldsymbol{x}\|_{\infty}\right), \\
\left\langle\boldsymbol{v}_{k}(t)-\boldsymbol{v}_{j}(t), \boldsymbol{v}_{k}(t)\right\rangle & \geq 0, \text { and } \\
\sum_{j=1}^{N}\left\langle\boldsymbol{v}_{k}(t)-\boldsymbol{v}_{j}(t), \boldsymbol{v}_{k}(t)\right\rangle & =N\left\|\boldsymbol{v}_{k}(t)\right\|^{2}-\left\langle\left(\sum_{j=1}^{N} \boldsymbol{v}_{j}(t)\right), \boldsymbol{v}_{k}(t)\right\rangle \\
& =N\left\|\boldsymbol{v}_{k}(t)\right\|^{2} \\
& =N\|\boldsymbol{v}(t)\|_{\infty}^{2} .
\end{aligned}
$$

This implies the desired differential inequality.

REMARK 3.1. Note that we only used the non-increasing property of $\psi$. Hence Lemma 3.1 can be applied in any situation as long as we have the solution in the time-interval $[0, T)$.

Following [22], we define two Lyapunov type functionals $\mathcal{E}_{ \pm}\left(\|\boldsymbol{x}\|_{\infty},\|\boldsymbol{v}\|_{\infty}\right)$ :

$$
\mathcal{E}_{ \pm}\left(\|\boldsymbol{x}(t)\|_{\infty},\|\boldsymbol{v}(t)\|_{\infty}\right):=\|\boldsymbol{v}(t)\|_{\infty} \pm \frac{K_{0}}{2} \Psi\left(2\|\boldsymbol{x}(t)\|_{\infty}\right),
$$

where $\Psi(\cdot)$ is an anti-derivative of $\psi$.

Lemma 3.2. Suppose (3.1) and (3.2) hold, and let $\left\{\left(\mathbf{x}_{i}, \boldsymbol{v}_{i}\right)\right\}_{i=1}^{N}$ be the global smooth solution to the system (2.1). Then for $t \in(0, \infty)$, we have

$$
\begin{aligned}
& \text { (i) } \mathcal{E}_{ \pm}\left(\|\boldsymbol{x}(t)\|_{\infty},\|\boldsymbol{v}(t)\|_{\infty}\right) \leq \mathcal{E}_{ \pm}\left(\left\|\boldsymbol{x}_{0}\right\|_{\infty},\left\|\boldsymbol{v}_{0}\right\|_{\infty}\right) \\
& \text { (ii) } \quad\|\boldsymbol{v}(t)\|_{\infty}+\frac{K_{0}}{2}\left|\int_{2\left\|\boldsymbol{x}_{0}\right\|_{\infty}}^{2\|\boldsymbol{x}(t)\|_{\infty}} \psi(s) d s\right| \leq\left\|\boldsymbol{v}_{0}\right\|_{\infty}
\end{aligned}
$$


Proof.

(i) We use Lemma 3.1 to get the following estimates:

$$
\begin{aligned}
& \frac{d}{d t} \mathcal{E}_{ \pm}\left(\|\boldsymbol{x}(t)\|_{\infty},\|\boldsymbol{v}(t)\|_{\infty}\right) \\
= & \frac{d}{d t}\left(\|\boldsymbol{v}(t)\|_{\infty} \pm \frac{K_{0}}{2} \Psi\left(2\|\boldsymbol{x}(t)\|_{\infty}\right)\right) \\
= & \frac{d\|\boldsymbol{v}(t)\|_{\infty}}{d t} \pm K_{0} \psi\left(2\|\boldsymbol{x}(t)\|_{\infty}\right) \frac{d\|\boldsymbol{x}(t)\|_{\infty}}{d t} \\
\leq & K_{0} \psi\left(2\|\boldsymbol{x}\|_{\infty}\right)\left(-\|\boldsymbol{v}\|_{\infty} \pm \frac{d\|\boldsymbol{x}\|_{\infty}}{d t}\right) \\
\leq & 0
\end{aligned}
$$

We integrate the above inequality to get the desired result.

(ii) It follows from the first inequality that

$$
\|\boldsymbol{v}(t)\|_{\infty}-\left\|\boldsymbol{v}_{0}\right\|_{\infty} \leq \pm \frac{K_{0}}{2}\left(\Psi\left(2\|\boldsymbol{x}(t)\|_{\infty}\right)-\Psi\left(2\left\|\boldsymbol{x}_{0}\right\|_{\infty}\right)\right) .
$$

Hence we have

$$
\begin{aligned}
\|\boldsymbol{v}(t)\|_{\infty}-\left\|\boldsymbol{v}_{0}\right\|_{\infty} & \leq-\frac{K_{0}}{2}\left|\Psi\left(2\|\boldsymbol{x}(t)\|_{\infty}\right)-\Psi\left(2\left\|\boldsymbol{x}_{0}\right\|_{\infty}\right)\right| \\
& =-\frac{K_{0}}{2}\left|\int_{2\left\|\boldsymbol{x}_{0}\right\|_{\infty}}^{2\|\boldsymbol{x}(t)\|_{\infty}} \psi(s) d s\right|
\end{aligned}
$$

and this completes the proof of the lemma.

As a direct application of Lemma 3.2, the asymptotic flocking estimates are obtained in the following theorem.

Theorem 3.3. Suppose (3.1) and (3.2) hold, and let $\left\{\left(\boldsymbol{x}_{i}, \boldsymbol{v}_{i}\right)\right\}_{i=1}^{N}$ be the global smooth solution to the system (2.1) with an initial configuration $\left\{\left(\boldsymbol{x}_{i 0}, \boldsymbol{v}_{i 0}\right)\right\}_{i=1}^{N}$ satisfying

$$
\left\|\boldsymbol{x}_{0}\right\|_{\infty}>0, \quad\left\|\boldsymbol{v}_{0}\right\|_{\infty}<\frac{K_{0}}{2} \min \left\{\int_{0}^{2\left\|\boldsymbol{x}_{0}\right\|_{\infty}} \psi(s) d s, \quad \int_{2\left\|\boldsymbol{x}_{0}\right\|_{\infty}}^{\infty} \psi(s) d s\right\} .
$$

Then we have

$$
0<x_{m} \leq\|\boldsymbol{x}(t)\|_{\infty} \leq x_{M}<\infty, \quad\|\boldsymbol{v}(t)\|_{\infty} \leq\left\|\boldsymbol{v}_{0}\right\|_{\infty} e^{-K_{0} \psi\left(2 x_{M}\right) t}, \quad t \geq 0 .
$$

Proof.

(i) Suppose that there exists $t_{*} \in(0, \infty)$ such that

$$
\left\|\boldsymbol{x}\left(t_{*}\right)\right\|_{\infty}<x_{m}
$$

and consider the second result of Lemma 3.2:

$$
\frac{K_{0}}{2}\left|\int_{2\left\|\boldsymbol{x}_{0}\right\|_{\infty}}^{2\|\boldsymbol{x}(t)\|_{\infty}} \psi(s) d s\right| \leq\left\|\boldsymbol{v}_{0}\right\|_{\infty}, \quad t \geq 0 .
$$


Since $\psi$ is positive and non-increasing, $\int_{\delta}^{2\left\|\boldsymbol{x}_{0}\right\|_{\infty}} \psi(s) d s$ is non-increasing on $\delta>0$. Therefore we can choose $x_{m}$ such that

$$
\left\|\boldsymbol{v}_{0}\right\|_{\infty}=\frac{K_{0}}{2} \int_{2 x_{m}}^{2\left\|\boldsymbol{x}_{0}\right\|_{\infty}} \psi(s) d s<\frac{K_{0}}{2} \int_{2\left\|\boldsymbol{x}\left(t_{*}\right)\right\|_{\infty}}^{2\left\|\boldsymbol{x}_{0}\right\|_{\infty}} \psi(s) d s .
$$

Hence, we have a contradiction, and we conclude that for any $t \geq 0$,

$$
x_{m} \leq\|\boldsymbol{x}(t)\|_{\infty} .
$$

(ii) We next show that

$$
\|\boldsymbol{x}(t)\|_{\infty} \leq x_{M}, \quad t \geq 0 .
$$

Suppose not, i.e., there exists $t_{*} \in(0, \infty)$ such that

$$
\left\|\boldsymbol{x}\left(t_{*}\right)\right\|_{\infty}>x_{M}
$$

On the other hand, it follows from Lemma 3.2 (ii) that

$$
\frac{K_{0}}{2}\left|\int_{2\left\|\boldsymbol{x}_{0}\right\|_{\infty}}^{2\|\boldsymbol{x}(t)\|_{\infty}} \psi(s) d s\right| \leq\left\|\boldsymbol{v}_{0}\right\|_{\infty}
$$

In particular, for $t=t_{*}$, we have

$$
\frac{K_{0}}{2}\left|\int_{2\left\|\boldsymbol{x}_{0}\right\|_{\infty}}^{2\left\|\boldsymbol{x}\left(t_{*}\right)\right\|_{\infty}} \psi(s) d s\right| \leq\left\|\boldsymbol{v}_{0}\right\|_{\infty} .
$$

However, with a similar argument to $(i)$, we can choose $x_{M}$ such that

$$
\left\|\boldsymbol{v}_{0}\right\|_{\infty}=\frac{K_{0}}{2} \int_{2\left\|\boldsymbol{x}_{0}\right\|_{\infty}}^{2 x_{M}} \psi(s) d s<\frac{K_{0}}{2}\left|\int_{2\left\|\boldsymbol{x}_{0}\right\|_{\infty}}^{2\left\|\boldsymbol{x}\left(t_{*}\right)\right\|_{\infty}} \psi(s) d s\right| .
$$

We combine (3.5) and (3.6) to derive the contradiction

$$
\left\|\boldsymbol{v}_{0}\right\|_{\infty}<\left\|\boldsymbol{v}_{0}\right\|_{\infty}
$$

(iii) We use the second inequality in (3.3), the first inequality, and the non-increasing property of $\psi$ to find the Gronwall-type inequality

$$
\frac{d\|\boldsymbol{v}\|_{\infty}}{d t} \leq-K_{0} \psi\left(2\|\boldsymbol{x}\|_{\infty}\right)\|\boldsymbol{v}\|_{\infty} \leq-K_{0} \psi\left(2 x_{M}\right)\|\boldsymbol{v}\|_{\infty} .
$$

This yields the desired result.

REMARK 3.2 .

1. A Lyapunov functional approach in the $\ell_{2}$-framework has been done in [22], however the results there depend on the number of particles $N$. Hence in the meanfield limit $(N \rightarrow \infty)$, the admissible class of initial data in [22] becomes trivial.

2. We remark that there is no finite-time collapse of particles in position. Suppose that all the particles collapse onto a one-point configuration at time $t_{*}$, i.e., $\boldsymbol{x}_{i}\left(t_{*}\right)=\boldsymbol{x}_{*}$ for all $i=1, \cdots, N$. On the other hand, it follows from (3.1) that

$$
0=\sum_{i=1}^{N} \boldsymbol{x}_{i}\left(t_{*}\right)=N \boldsymbol{x}_{*} .
$$


Therefore we know that $\boldsymbol{x}_{*}=0$ and it implies that

$$
\left\|\boldsymbol{x}\left(t_{*}\right)\right\|_{\infty}=0 .
$$

However, the Theorem 3.3 shows that it is not possible since

$$
0<x_{m} \leq\left\|\boldsymbol{x}\left(t_{*}\right)\right\|_{\infty}
$$

Therefore there is no finite-time collapse to a one-point configuration.

\section{A global existence theory for the C-S model with singular weights}

In this section, we study the global existence of the C-S model with singular communication as below.

Let $\psi:[0, \infty) \rightarrow[0, \infty]$ be a continuous function satisfying

- $(\mathcal{A} 1) \psi$ is non-increasing and decays to zero as $r \rightarrow \infty$ :

$$
\begin{aligned}
& \left(\psi\left(r_{2}\right)-\psi\left(r_{1}\right)\right)\left(r_{2}-r_{1}\right) \leq 0, \quad r_{1}, r_{2}>0 \\
& \psi(r)>0, \quad r \geq 0, \quad \lim _{r \rightarrow \infty} \psi(r)=0 .
\end{aligned}
$$

- $(\mathcal{A} 2) \psi$ is singular at $r=0$ :

$$
\int_{0}^{\delta} \psi(s) d s=\infty, \quad \text { for any } \delta>0 .
$$

Note that the long-ranged weight $\psi(r)=r^{-\alpha}, \alpha \geq 1$ satisfies the above assumptions, and it follows from $(\mathcal{A} 2)$ that we have

$$
\lim _{r \rightarrow 0+} \psi(r)=\infty .
$$

Therefore when the particles collide, the force becomes infinity, and the standard existence theory is not applicable for this case. Hence we cannot have a global smooth solution to the system (2.1) in the presence of collisions. Therefore in order to obtain the global smooth solutions, we need to restrict our admissible initial data. In the sequel, we will try to find initial configurations which prevent collisions in finite-time so that we can have a global existence of smooth solutions for this kind of initial data.

4.1. A two-particle system. In this part, we consider two-particles on a line. All the positions and velocities in the two-particle system are assumed to be on the same line, i.e., $x_{i}, v_{i} \in \mathbb{R}, i=1,2$. The system (2.1) for a two-particle system reads as follows:

$$
\begin{array}{ll}
\frac{d x_{1}}{d t}=v_{1}, & \frac{d v_{1}}{d t}=\frac{K_{0}}{2} \psi\left(r_{12}\right)\left(v_{2}-v_{1}\right), \\
\frac{d x_{2}}{d t}=v_{2}, & \frac{d v_{2}}{d t}=\frac{K_{0}}{2} \psi\left(r_{21}\right)\left(v_{1}-v_{2}\right),
\end{array}
$$

subject to initial data

$$
\left(x_{i}(0), v_{i}(0)\right)=\left(x_{i 0}, v_{i 0}\right) .
$$

To reduce the number of equations, we set

$$
x:=x_{1}-x_{2} \quad \text { and } \quad v:=v_{1}-v_{2} .
$$


Then $x$ and $v$ satisfy the following system of ODEs:

$$
\frac{d x}{d t}=v, \quad \frac{d v}{d t}=-K_{0} \psi(|x|) v,
$$

and this also implies

$$
d v=-K_{0} \psi(|x|) d x .
$$

We integrate the above Equation (4.6) to find

$$
v(t)+K_{0} \int_{x_{0}}^{x(t)} \psi(r) d r=v_{0}, \quad t>0 .
$$

For given $x_{0} \neq 0$, we set the maximal life-span $T\left(x_{0}\right)$ of the initial datum $x_{0}$ as follows: $T\left(x_{0}\right):=\sup \{\tau \in \mathbb{R}: \exists$ solution $(x(t), v(t))$ to the system (4.5) in time-interval $[0, \tau)\}$.

Note that as long as there is no collision, the solution can be defined. Hence $T\left(x_{0}\right)<\infty$ means that the solution $x(t)$ becomes zero at time $t=T\left(x_{0}\right)$, i.e.,

$$
\lim _{t \rightarrow T\left(x_{0}\right)-} x(t)=0
$$

Proposition 4.1. (No finite-time collisions). Suppose the communication weight $\psi$ satisfies the assumptions $(\mathcal{A} 1)$ and $(\mathcal{A} 2)$ in Section 2.3. Then for any $x_{0} \neq 0$, we have

$$
T\left(x_{0}\right)=\infty,
$$

i.e., there exists a global solution $(x(t), v(t))$ to the system (4.5).

Proof. Let $x_{0} \neq 0$. Without loss of generality, we assume

$$
x_{0}>0 \text {. }
$$

Then the proof follows from (4.7) and the estimate

$$
\sup _{t>0}|v(t)| \leq\left|v_{0}\right|
$$

For the proof of (4.8), we multiply the second equation of (4.5) by $2 v$ to find

$$
\frac{d|v|^{2}}{d t}=-2 K_{0} \psi(|x|)|v|^{2} \leq 0
$$

This yields the desired estimate (4.8). We now return to the proof. Suppose that the maximal life-span of $x_{0}$ is finite, say

$$
T\left(x_{0}\right)<\infty .
$$

Then we let $t \nearrow T\left(x_{0}\right)$ in the relation (4.7) and use $x\left(T\left(x_{0}\right)\right)=0$ to get the contradiction:

$$
\infty=\left|K_{0} \int_{x_{0}}^{x\left(T\left(x_{0}\right)\right)} \psi(r) d r\right|=\left|K_{0} \int_{0}^{x_{0}} \psi(r) d r\right|=\left|v_{0}-v\left(T\left(x_{0}\right)\right)\right| \leq 2\left|v_{0}\right|<\infty .
$$


Therefore we have

$$
T\left(x_{0}\right)=\infty
$$

Corollary 4.2. Let $(x(t), v(t))$ be a global smooth solution to the system (4.5) with initial data $\left(x_{0}, v_{0}\right)$ satisfying

$$
\left|x_{0}\right|>0, \quad\left|v_{0}\right|<K_{0} \int_{\left|x_{0}\right|}^{\infty} \psi(s) d s,
$$

then the asymptotic flocking occurs. More precisely, there exist positive constant $x_{M}>$ 0 such that

$$
\sup _{t \geq 0}|x(t)| \leq x_{M}, \quad|v(t)| \leq\left|v_{0}\right| e^{-K_{0} \psi\left(x_{M}\right) t}, \quad t \geq 0,
$$

where $x_{M}$ is uniquely defined by the following implicit relation:

$$
\left|v_{0}\right|=K_{0} \int_{\left|x_{0}\right|}^{x_{M}} \psi(s) d s .
$$

Proof. The proof is exactly the same as Theorem 3.1.

REMARK 4.1. When the asymptotic flocking occurs, it follows from (4.7) that the asymptotic spatial difference $x_{\infty}=\lim _{t \rightarrow \infty} x(t)$ is implicitly determined by the following relation:

$$
K_{0} \int_{x_{0}}^{x_{\infty}} \psi(s) d s=v_{0}
$$

We next consider the $N$-particle system with $N \geq 3$.

4.2. A many-body particle system. In this part, we present a set of initial configurations leading to no finite-time collisions. Hence the global solutions can be launched from this initial data regardless of singularity of communication weights. For a given $N$-particle configuration $\left\{\left(\boldsymbol{x}_{i}, \boldsymbol{v}_{i}\right)\right\}_{i=1}^{N}$, we set

$$
X_{i j}:=\left\|\boldsymbol{x}_{i}-\boldsymbol{x}_{j}\right\|, \quad V_{i j}:=\left\|\boldsymbol{v}_{i}-\boldsymbol{v}_{j}\right\| .
$$

Lemma 4.3. Suppose the assumptions $(\mathcal{A} 1)-(\mathcal{A} 2)$ hold, and for a given $T \in(0, \infty]$, let $\left\{\left(\boldsymbol{x}_{i}, \boldsymbol{v}_{i}\right)\right\}_{i=1}^{N}$ be the solution to the (2.1) in the time-interval $[0, T)$ with an initial configuration $\left\{\left(\boldsymbol{x}_{i 0}, \boldsymbol{v}_{i 0}\right)\right\}_{i=1}^{N}$. Then we have

$$
\frac{d X_{i j}(t)}{d t} \leq 2\left\|\boldsymbol{v}_{0}\right\|_{\infty} e^{-K_{0} \psi\left(2 x_{M}\right) t}, \quad \text { a.e. } t \in[0, T),
$$

where $x_{M}$ is defined implicitly by the following relation:

$$
\left\|\boldsymbol{v}_{0}\right\|_{\infty}=\frac{K_{0}}{2} \int_{2\left\|\boldsymbol{x}_{0}\right\|_{\infty}}^{2 x_{M}} \psi(s) d s .
$$

Proof. By direct calculation, we have 


$$
\frac{d X_{i j}^{2}}{d t}=2\left\langle\boldsymbol{x}_{i}-\boldsymbol{x}_{j}, \boldsymbol{v}_{i}-\boldsymbol{v}_{j}\right\rangle \leq 2\left\|\boldsymbol{x}_{i}-\boldsymbol{x}_{j}\right\|\left\|\boldsymbol{v}_{i}-\boldsymbol{v}_{j}\right\|=2 X_{i j} V_{i j}
$$

This implies

$$
\frac{d X_{i j}}{d t} \leq V_{i j}
$$

On the other hand, note that Lemma 3.1 can be applied for any local smooth solution, i.e., we have

$$
\frac{d\|\boldsymbol{v}\|_{\infty}}{d t} \leq-K_{0} \psi\left(2\|\boldsymbol{x}\|_{\infty}\right)\|\boldsymbol{v}\|_{\infty} \leq-K_{0} \psi\left(2 x_{M}\right)\|\boldsymbol{v}\|_{\infty},
$$

where we have used the fact $\|\boldsymbol{x}(t)\|_{\infty} \leq x_{M}$ and the non-increasing property of $\psi$. Then Gronwall's lemma yields

$$
\|\boldsymbol{v}\|_{\infty} \leq\left\|\boldsymbol{v}_{0}\right\|_{\infty} e^{-K_{0} \psi\left(2 x_{M}\right) t}
$$

We finally combine (4.9) and (4.10) to get

$$
\frac{d X_{i j}}{d t} \leq V_{i j} \leq\left\|\boldsymbol{v}_{i}\right\|+\left\|\boldsymbol{v}_{j}\right\| \leq 2\|\boldsymbol{v}\|_{\infty} \leq 2\left\|\boldsymbol{v}_{0}\right\|_{\infty} e^{-K_{0} \psi\left(2 x_{M}\right) t} .
$$

We now define a set consisting of all admissible initial configurations leading to no finite-time collisions. For this, we define

$$
\mathcal{S}_{1}:=\left\{\left\{\left(\boldsymbol{x}_{i 0}, \boldsymbol{v}_{i 0}\right)\right\}_{i=1}^{N} \in \mathbb{R}^{2 d N}: d_{m}\left(\boldsymbol{x}_{0}, \boldsymbol{v}_{0}\right):=\min _{1 \leq i \neq j \leq N}\left|X_{i j}(0)\right|-\frac{2|| \boldsymbol{v}_{0} \|_{\infty}}{K_{0} \psi\left(2 x_{M}\right)}>0\right\} .
$$

THEOREM 4.4. Suppose the assumptions $(\mathcal{A} 1)-(\mathcal{A} 2)$ holds and the initial data $\left\{\left(\boldsymbol{x}_{i 0}, \boldsymbol{v}_{i 0}\right)\right\}_{i=1}^{N}$ lies in the set $\mathcal{S}_{1}$. Then there exists a unique global smooth solution $\left\{\left(\boldsymbol{x}_{i}, \boldsymbol{v}_{i}\right)\right\}_{i=1}^{N}$ to the system (2.1) satisfying

$$
\inf _{t \geq 0} \min _{i, j} X_{i j}(t) \geq d_{m}\left(\boldsymbol{x}_{0}, \boldsymbol{v}_{0}\right), \quad t \geq 0 .
$$

Proof. Since the initial data $\left\{\left(\boldsymbol{x}_{i 0}, \boldsymbol{v}_{i 0}\right)\right\}_{i=1}^{N}$ is in the set $\mathcal{S}_{1}$, we have

$$
d_{m}\left(\boldsymbol{x}_{0}, \boldsymbol{v}_{0}\right):=\min _{1 \leq i \neq j \leq N}\left|X_{i j}(0)\right|-\frac{2\left\|\boldsymbol{v}_{0}\right\|_{\infty}}{K_{0} \psi\left(2 x_{M}\right)}>0 .
$$

As long as the minimal distance between particle is away from zero, i.e., there is no finite-time collisions between particles, we will have the global existence. Hence once we establish

$$
\inf _{t \geq 0} \min _{i, j} X_{i j}(t) \geq d_{m}\left(\boldsymbol{x}_{0}, \boldsymbol{v}_{0}\right)>0,
$$

we will have the global existence of a smooth solution. For the estimate of (4.11), we use Lemma 4.3 to find

$$
\begin{aligned}
\left|X_{i j}(t)-X_{i j}(0)\right| & \leq\left|\int_{0}^{t} \frac{d X_{i j}(s)}{d s} d s\right| \leq \int_{0}^{t}\left|V_{i j}(s)\right| d s \\
& \leq 2\left\|\boldsymbol{v}_{0}\right\|_{\infty} \int_{0}^{t} e^{-K_{0} \psi\left(2 x_{M}\right) s} d s \\
& =\frac{2\left\|\boldsymbol{v}_{0}\right\|_{\infty}}{K_{0} \psi\left(2 x_{M}\right)}\left(1-e^{-K_{0} \psi\left(2 x_{M}\right) t}\right) \leq \frac{2\left\|\boldsymbol{v}_{0}\right\|_{\infty}}{K_{0} \psi\left(2 x_{M}\right)} .
\end{aligned}
$$


Then we obtain the desired result by the triangle inequality as follows:

$$
\left|X_{i j}(t)\right| \geq\left|X_{i j}(0)\right|-\left|X_{i j}(t)-X_{i j}(0)\right| \geq\left|X_{i j}(0)\right|-\frac{2|| \boldsymbol{v}_{0} \|_{\infty}}{K_{0} \psi\left(2 x_{M}\right)} .
$$

As a corollary of Theorem 4.1, we obtain the asymptotic flocking for configurations in $\mathcal{S}$.

Corollary 4.5. For the global solutions $\left\{\left(\mathbf{x}_{i}, \boldsymbol{v}_{i}\right)\right\}_{i=1}^{N}$ constructed in Theorem 4.1, we have asymptotic flocking: For $t \in(0, \infty)$, we have

$$
\sup _{t \geq 0}\left|X_{i j}(t)\right| \leq\left|X_{i j}(0)\right|+\frac{2\left\|\boldsymbol{v}_{0}\right\|_{\infty}}{K_{0} \psi\left(2 x_{M}\right)} \quad \text { and } \quad \lim _{t \rightarrow \infty} V_{i j}(t)=0, \quad i, j \in\{1, \cdots, N\} \text {. }
$$

Proof. The estimates follow from the relation (4.12) and (4.10).

\section{A global existence for the C-S model with a bonding force}

In this section, we present a global existence theory for the Cucker-Smale flocking model (2.3) with a bonding force [29] for some class of initial configurations. Recall that the Cucker-Smale flocking model with a bonding force reads as follows:

$$
\begin{aligned}
\frac{d \boldsymbol{x}_{i}}{d t}= & \boldsymbol{v}_{i}, \\
\frac{d \boldsymbol{v}_{i}}{d t}= & \frac{K_{0}}{N} \sum_{\substack{j \neq i \\
j=1}}^{N} \psi\left(r_{i j}\right)\left(\boldsymbol{v}_{j}-\boldsymbol{v}_{i}\right)+\frac{K_{1}}{N} \sum_{\substack{j \neq i \\
j=1}}^{N} \frac{1}{2 r_{i j}^{2}}\left\langle\boldsymbol{v}_{i}-\boldsymbol{v}_{j}, \boldsymbol{x}_{i}-\boldsymbol{x}_{j}\right\rangle\left(\boldsymbol{x}_{j}-\boldsymbol{x}_{i}\right) \\
& +\frac{K_{2}}{N} \sum_{\substack{j \neq i \\
j=1}}^{N} \frac{1}{2 r_{i j}}\left(r_{i j}-2 R\right)\left(\boldsymbol{x}_{j}-\boldsymbol{x}_{i}\right),
\end{aligned}
$$

where the constants $K_{i}, i=0,1,2$ denote the coupling strengths; we refer to [29] for the derivation of the system. Since the total momentum is conserved along the flow, without loss of generality we set

$$
\sum_{i=1}^{N} \boldsymbol{x}_{i}=0, \quad \sum_{i=1}^{N} \boldsymbol{v}_{i}=0, \quad t \geq 0 .
$$

5.1. A priori estimates. In this part, we summarize a priori estimates from [29]. We introduce the kinetic energy $E_{k}$, the configuration energy $E_{c}$, and the total energy $E$ :

$$
E_{k}:=\frac{1}{2} \sum_{i=1}^{N}\left\|\boldsymbol{v}_{i}\right\|^{2}, \quad E_{c}:=\frac{K_{2}}{8 N} \sum_{i, j=1}^{N}\left(r_{i j}-2 R\right)^{2}, \quad E=E_{k}+E_{c} .
$$

The flocking estimate results in [29] were obtained by the energy estimates. In the sequel, we present the main estimates in [29] without the proofs.

Proposition 5.1. [29] Let $\left\{\left(\mathbf{x}_{i}, \boldsymbol{v}_{i}\right)\right\}_{i=1}^{N}$ be the global solution to the system (2.3). Then $E=E(t)$ is non-increasing in time $t$ :

$$
E(t)+\int_{0}^{t} P(\tau) d \tau=E(0), \quad t \geq 0
$$


where $P$ is the total energy production rate of the system:

$$
P:=\frac{K_{0}}{2 N} \sum_{\substack{i \neq j \\ i, j=1}}^{N} \psi\left(r_{i j}\right)\left\|\boldsymbol{v}_{i}-\boldsymbol{v}_{j}\right\|^{2}+\frac{K_{1}}{4 N} \sum_{\substack{i \neq j \\ i, j=1}}^{N}\left(\frac{d r_{i j}}{d t}\right)^{2} .
$$

Using the above energy estimate gives the following two propositions.

Proposition 5.2. [29] Suppose $\psi$ satisfies the assumptions $(\mathcal{A} 1)-(\mathcal{A} 2)$, and let $\left\{\left(\mathbf{x}_{i}, \boldsymbol{v}_{i}\right)\right\}_{i=1}^{N}$ be the global smooth solutions to the system (2.3). Then the system (2.3) exhibits asymptotic flocking estimates: For $t \geq 0$,

$$
\begin{aligned}
& \text { (i) } \sup _{0 \leq t<\infty}\left\|\boldsymbol{x}_{i}(t)-\boldsymbol{x}_{j}(t)\right\|<2 R+\sqrt{\frac{4 N E(0)}{K_{2}}} . \\
& \text { (ii) } \lim _{t \rightarrow \infty}\left\|\boldsymbol{v}_{i}(t)-\boldsymbol{v}_{j}(t)\right\|=0, \quad 1 \leq i, j \leq N .
\end{aligned}
$$

Proposition 5.3. [29] Let $\left\{\left(\mathbf{x}_{i}, \boldsymbol{v}_{i}\right)\right\}_{i=1}^{N}$ be the global smooth solutions to the system (2.3) satisfying the condition

$$
E(0)<\frac{K_{2}}{2} R^{2} N
$$

Then the particle system does not collapse to a one-point configuration at any finitetime.

Proof. We refer to [29] for the proof.

As we can see from the statement of the Theorem 4.1, the solutions $\left\{\left(\boldsymbol{x}_{i}, \boldsymbol{v}_{i}\right)\right\}_{i=1}^{N}$ exist on the whole time interval $[0, \infty)$. The existence of solutions to the system $(2.3)$ follows from the well-known theory of ODEs as long as the right hand side of (2.3) is Lipschitz continuous. We note that the Lipschitz continuity of the right hand side of (2.3) is sufficiently guaranteed by the collision avoidance between particles, i.e., $r_{i j}(t) \neq 0$ for any $t \geq 0$ and $i \neq j$. In order to show the collision avoidance, we need to introduce a new quantity.

5.2. A new spatial fluctuation functional. In this part, we introduce a new functional measuring the degree of fluctuations around the equilibria to the system (2.3). We next present a heuristic argument for the derivation of the functional as follows. Suppose that sufficiently strong flocking occurs asymptotically, so that

$$
\lim _{t \rightarrow \infty} \boldsymbol{v}_{i}=0, \quad \lim _{t \rightarrow \infty} \dot{\boldsymbol{v}}_{i}=0, \quad i=1, \cdots, N .
$$

Then it follows from (5.2) and (2.3) that

$$
\begin{aligned}
\mathbf{0} & =\lim _{t \rightarrow \infty} \sum_{\substack{j \neq i \\
j=1}}^{N}\left[\left(r_{i j}-2 R\right) \frac{\boldsymbol{x}_{j}-\boldsymbol{x}_{i}}{r_{i j}}\right] \\
& =\lim _{t \rightarrow \infty}\left[\sum_{\substack{j \neq i \\
j=1}}^{N}\left(\boldsymbol{x}_{j}-\boldsymbol{x}_{i}\right)-2 R \sum_{\substack{j \neq i \\
j=1}}^{N} \frac{\boldsymbol{x}_{j}-\boldsymbol{x}_{i}}{r_{i j}}\right] \\
& =-N \lim _{t \rightarrow \infty}\left[\boldsymbol{x}_{i}+\frac{2 R}{N} \sum_{\substack{j \neq i \\
j=1}}^{N} \frac{\boldsymbol{x}_{j}-\boldsymbol{x}_{i}}{r_{i j}}\right], \quad \text { using } \quad \sum_{i=1}^{N} \boldsymbol{x}_{i}=0 .
\end{aligned}
$$


We now set

$$
\mathbf{D}_{i}:=\boldsymbol{x}_{i}-\frac{2 R}{N} \sum_{\substack{j \neq i \\ j=1}}^{N} \frac{\boldsymbol{x}_{i}-\boldsymbol{x}_{j}}{r_{i j}} .
$$

Then the quantity $\mathbf{D}_{i}$ measures the spatial distance from the $i$-th particle to the flocking state. We now define a new functional $E_{p}$ as the square of $\ell_{2}$-norm of $\mathbf{D}=$ $\left(\mathbf{D}_{1}, \cdots, \mathbf{D}_{N}\right)$ :

$$
E_{p}:=\sum_{i=1}^{N}\left\|\mathbf{D}_{i}\right\|^{2}
$$

In the following lemma, we will see that as long as the initial fluctuation energy $E(0)$ is finite, $E_{p}$ is finite, and hence is a well-defined object, then when the flocking occurs asymptotically, $E_{p}$ goes to zero as $t \rightarrow \infty$, but the configuration energy $E_{c}$ does not converge to zero. Hence in some sense, the functional $E_{p}$ measures the discrepancy from the flocking state. In the following lemma, we will see that the functional $E_{p}$ is dominated by $E_{c}$.

Lemma 5.4. Let $E_{p}$ be the spatial fluctuation energy associated with the system (2.3). Then we have

$$
E_{p}(t) \leq \frac{8}{N K_{2}} E(0), \quad t \geq 0
$$

Proof. It is sufficient to show that $E_{p}$ is bounded by $E_{c}$. By direct calculation, we have

$$
\begin{aligned}
E_{p} & =\sum_{i=1}^{N}\left\|\boldsymbol{x}_{i}-\frac{2 R}{N} \sum_{j \neq i} \frac{\boldsymbol{x}_{i}-\boldsymbol{x}_{j}}{r_{i j}}\right\|^{2}=\frac{1}{N^{2}} \sum_{i=1}^{N}\left\|\sum_{j \neq i}\left(r_{i j}-2 R\right) \frac{\boldsymbol{x}_{i}-\boldsymbol{x}_{j}}{r_{i j}}\right\|^{2} \\
& \leq \frac{1}{N^{2}} \sum_{i=1}^{N} \sum_{j \neq i}\left(r_{i j}-2 R\right)^{2} \leq \frac{1}{N^{2}} \sum_{i, j=1}^{N}\left(r_{i j}-2 R\right)^{2}=\frac{8}{N K_{2}} E_{c} .
\end{aligned}
$$

We now use

$$
E_{c}(t) \leq E(t) \leq E(0), \quad t \geq 0
$$

to get the desired result.

REMARK 5.1. Note that when the flocking occurs sufficiently fast, the particle system is in an equilibrium state. In the equilibrium configuration, the position of each particle is determined by the relative positions with the other particles:

$$
\boldsymbol{x}_{i}^{\infty}=\frac{2 R}{N} \sum_{\substack{j \neq i \\ j=1}}^{N} \frac{\boldsymbol{x}_{i}^{\infty}-\boldsymbol{x}_{j}^{\infty}}{r_{i j}^{\infty}}, \quad \boldsymbol{x}_{i}^{\infty}:=\lim _{t \rightarrow \infty} \boldsymbol{x}_{i}, \quad r_{i j}^{\infty}:=\left\|\boldsymbol{x}_{i}^{\infty}-\boldsymbol{x}_{j}^{\infty}\right\| .
$$

This yields

$$
\left\|\boldsymbol{x}_{i}^{\infty}\right\| \leq \frac{2 R(N-1)}{N}<2 R
$$


Hence all particles are strictly confined in $B\left(\boldsymbol{x}_{c}, 2 R\right)$ regardless of the asymptotic size of the system.

We now study the structure of the functional $E_{p}$ :

$$
\begin{aligned}
E_{p}= & \sum_{i=1}^{N}\left\|\boldsymbol{x}_{i}-\frac{2 R}{N} \sum_{j \neq i} \frac{\boldsymbol{x}_{i}-\boldsymbol{x}_{j}}{r_{i j}}\right\|^{2} \\
= & \sum_{i=1}^{N}\left\|\boldsymbol{x}_{i}\right\|^{2}-\frac{4 R}{N} \sum_{i=1}^{N} \sum_{j \neq i}\left\langle\boldsymbol{x}_{i}, \frac{\boldsymbol{x}_{i}-\boldsymbol{x}_{j}}{r_{i j}}\right\rangle \\
& +\frac{4 R^{2}}{N^{2}} \sum_{i=1}^{N} \sum_{j \neq i} \sum_{k \neq i}\left\langle\frac{\boldsymbol{x}_{i}-\boldsymbol{x}_{j}}{r_{i j}}, \frac{\boldsymbol{x}_{i}-\boldsymbol{x}_{k}}{r_{i k}}\right\rangle \\
= & \sum_{i=1}^{N}\left\|\boldsymbol{x}_{i}\right\|^{2}-\frac{4 R}{N} \mathcal{I}_{1}+\frac{4 R^{2}}{N^{2}} \mathcal{I}_{2} .
\end{aligned}
$$

In the following lemma, we estimate $\mathcal{I}_{i}, i=1,2$.

Lemma 5.5. The terms $\mathcal{I}_{i}, i=1,2$ satisfy the following estimates.

$$
\mathcal{I}_{1}=\frac{1}{2} \sum_{i=1}^{N} \sum_{j \neq i} r_{i j} \quad \text { and } \quad \mathcal{I}_{2}=\frac{1}{2} \sum_{i=1}^{N} \sum_{j \neq i} \sum_{k \neq i} \frac{r_{i j}}{r_{i k}} .
$$

Proof.

(i) We use the standard symmetrization trick $i \leftrightarrow j$ and $\left\|\boldsymbol{x}_{i}-\boldsymbol{x}_{j}\right\|=r_{i j}$ to see

$$
\begin{aligned}
\mathcal{I}_{1} & =\sum_{i=1}^{N} \sum_{j \neq i}\left\langle\boldsymbol{x}_{i}, \frac{\boldsymbol{x}_{i}-\boldsymbol{x}_{j}}{r_{i j}}\right\rangle=-\sum_{i=1}^{N} \sum_{j \neq i}\left\langle\boldsymbol{x}_{j}, \frac{\boldsymbol{x}_{i}-\boldsymbol{x}_{j}}{r_{i j}}\right\rangle \\
& =\frac{1}{2} \sum_{i=1}^{N} \sum_{j \neq i}\left\langle\boldsymbol{x}_{i}-\boldsymbol{x}_{j}, \frac{\boldsymbol{x}_{i}-\boldsymbol{x}_{j}}{r_{i j}}\right\rangle=\frac{1}{2} \sum_{i=1}^{N} \sum_{j \neq i} r_{i j},
\end{aligned}
$$

(ii) Similar to the case (i), we have

$$
\sum_{i=1}^{N} \sum_{j \neq i} \sum_{k \neq i}\left\langle\frac{\boldsymbol{x}_{i}-\boldsymbol{x}_{j}}{r_{i j}}, \frac{\boldsymbol{x}_{i}-\boldsymbol{x}_{k}}{r_{i k}}\right\rangle=\frac{1}{2} \sum_{i=1}^{N} \sum_{j \neq i} \sum_{k \neq i} \frac{r_{i j}}{r_{i k}} .
$$

5.3. Existence of global smooth solutions. We now discuss the collision avoidance between particles. In [29], the authors found a condition on initial data which prevents the particles from collapsing to a one-point configuration. Note that the fact that the system does not collapse to a one-point configuration does not imply that the particles in the system do not collide with each other. However, under the same condition on initial energy as in Proposition 5.3, we show that there are no collisions between particles. We now introduce a set of initial configurations leading to global existence:

$$
\mathcal{S}_{2}:=\left\{(\boldsymbol{x}, \boldsymbol{v}) \in \mathbb{R}^{2 d N}: E(\boldsymbol{x}, \boldsymbol{v})<\frac{K_{2}}{2} R^{2} N, \quad \min _{i \neq j}\left\|\boldsymbol{x}_{i}-\boldsymbol{x}_{j}\right\|>0\right\} .
$$


Proposition 5.6. Suppose the main assumptions $(\mathcal{A} 1)-(\mathcal{A} 2)$ on $\psi$ in Section 2.3 hold and the initial configuration lies in the set $\mathcal{S}_{2}$. For some $T \in(0, \infty]$, let $\left\{\left(\boldsymbol{x}_{i}, \boldsymbol{v}_{i}\right)\right\}_{i=1}^{N}$ be the smooth solution to the system (2.3) in the time interval $[0, T)$. Then the maximal and minimal inter-particle distances are comparable in the sense that

$$
\max _{j \neq i} r_{i j}(t) \leq \sqrt{2} N^{3}(N+\sqrt{2}-1) \min _{k \neq i} r_{i k}(t), \quad i=1, \cdots, N, \quad t \in[0, T),
$$

where $r_{i j}=\left\|\boldsymbol{x}_{i}-\boldsymbol{x}_{j}\right\|$.

Proof. We use (5.3) and Lemma 5.5 to obtain

$$
E_{p} \geq-\frac{2 R}{N} \sum_{i=1}^{N} \sum_{j \neq i} r_{i j}+\frac{2 R^{2}}{N^{2}} \sum_{i=1}^{N} \sum_{j \neq i} \sum_{k \neq i} \frac{r_{i j}}{r_{i k}} .
$$

We now apply the boundedness of $E_{p}$ in Lemma 5.4 to the left hand side and Proposition 5.2 to the first term of the right hand side. Then we have

$$
\frac{8}{N K_{2}} E(0)+2 R(N-1)\left(2 R+\sqrt{\frac{4 N E(0)}{K_{2}}}\right) \geq \frac{2 R^{2}}{N^{2}} \sum_{i=1}^{N} \sum_{j \neq i} \sum_{k \neq i} \frac{r_{i j}}{r_{i k}} .
$$

We finally apply the condition on the initial energy $E(0)$, which is the same as the one in Proposition 5.3, to the left hand side to obtain

$$
4 R^{2}+2 R(N-1)\left(2 R+\sqrt{2 R^{2} N^{2}}\right) \geq \frac{2 R^{2}}{N^{2}} \sum_{i=1}^{N} \sum_{j \neq i} \sum_{k \neq i} \frac{r_{i j}}{r_{i k}} .
$$

Since each of the $r_{i j}$ in the right hand side is positive, we obtain for any $i, j, k$ with $i \neq j$ and $i \neq k$,

$$
\begin{aligned}
\frac{r_{i j}}{r_{i k}} & \leq \frac{N^{2}}{2 R^{2}}\left[4 R^{2}+2 R(N-1)\left(2 R+\sqrt{2 R^{2} N^{2}}\right)\right] \\
& =N^{2}(2+(N-1)(2+\sqrt{2} N)) \\
& =N^{3}(\sqrt{2} N+2-\sqrt{2}),
\end{aligned}
$$

or equivalently for any $i, j, k$ with $i \neq j$ and $i \neq k$,

$$
r_{i j} \leq \sqrt{2} N^{3}(N+\sqrt{2}-1) r_{i k} .
$$

We take the maximum on $j$ and minimum on $k$ to obtain

$$
\max _{j \neq i} r_{i j} \leq \sqrt{2} N^{3}(N+\sqrt{2}-1) \min _{k \neq i} r_{i k}, \quad i=1, \cdots, N .
$$

We now ready to prove global existence for some class of initial configurations.

Theorem 5.7. Suppose the main assumptions $(\mathcal{A} 1)-(\mathcal{A} 2)$ on $\psi$ in Section 2.3 hold and the initial configuration $\left\{\left(\mathbf{x}_{i 0}, \boldsymbol{v}_{i 0}\right)\right\}_{i=1}^{N}$ lies in the set $\mathcal{S}_{2}$. Then there exists a global smooth solution $\left\{\left(\mathbf{x}_{i}, \boldsymbol{v}_{i}\right)\right\}_{i=1}^{N}$ to the system (2.3). 
Proof. Let $\left\{\left(\mathbf{x}_{i 0}, \boldsymbol{v}_{i 0}\right)\right\}_{i=1}^{N} \in \mathcal{S}_{2}$, and let $T_{*}$ be the maximal-time when the local solution can be constructed in the time-interval $\left[0, T_{*}\right)$. If we can show $T_{*}=\infty$, then we are done. Suppose $T_{*}<\infty$. That means that there exist $i \neq j \in\{1, \cdots, N\}$ such that

$$
\boldsymbol{x}_{i}\left(T_{*}-\right)=\boldsymbol{x}_{j}\left(T_{*}-\right) .
$$

Then it follows from Proposition 5.6 that

$$
\boldsymbol{x}_{i}\left(T_{*}-\right)=\boldsymbol{x}_{j}\left(T_{*}-\right), \quad \forall i, j \in\{1, \cdots, N\},
$$

i.e., all particles collapse to one-point at time $t=T_{*}$, which gives the contradiction to Proposition 5.3. Therefore we have $T_{*}=\infty$.

\section{Conclusion}

We presented a global existence and asymptotic flocking estimates for the CuckerSmale type models with singular communication weights and inter-particle bonding forces. We discussed three issues in this paper. First we presented a $\ell_{\infty}$-Lyapunov functional approach for some general class of regular communication weights combining the $\ell_{2}$-Lyapunov functional approach and Carrillo et al's $\ell_{\infty}$ approach for the special Cucker-Smale communication weights. Our result is independent of the number of particles, hence it can be lifted to the kinetic regime via the mean-field limit. Secondly, we presented a global existence theory for the Cucker-Smale flocking model with singular communication weights. The forcing term becomes singular when two particles collide. We explicitly identified initial configurations that do not lead to pairwise collisions in a finite-time. So for this class of initial configurations, we have the global existence of smooth solutions even for the singular forcing terms. Thirdly, we presented a global existence of smooth solutions to the Cucker-Smale model with inter-particle bonding forces. In [29], a priori asymptotic flocking estimates were given without any specific decay rates, and exclusion of pairwise collisions between particles was not treated, hence for some initial configurations, the finite-time collisions can occur. However we showed that when the initial energy is bounded by some predetermined upper bound and initially all particles are away from each other, the finite-time pairwise collisions are not possible. Hence those initial configurations launch the global smooth solutions. In previous literature, there were recent studies to prevent collisions for the Cucker-Smale model by adding some extra collision avoidance mechanisms. The main message of this paper is that the singular communication weights and some well-chosen initial configurations do not undergo the finite-time pairwise collisions for the original Cucker-Smale flocking model without any extra collision avoidance mechanisms.

Acknowledgment. The work of S.-Y. Ha was supported by the research grant of Korea Research Foundation (KRF 2008-C00060) and research grant of SNU-CNS.

\section{REFERENCES}

[1] S. Ahn and S.Y. Ha, Stochastic flocking dynamics of the Cucker-Smale model with multiplicative white noises, J. Math. Phys., 51, 103301, 2010.

[2] F. Bolley, J.A. Canizo, and J.A. Carrillo, Stochastic mean-field limit: Non-Lipschitz forces and swarming, Math. Mod. Meth. Appl. Sci., to appear.

[3] J.A. Canizo, J.A. Carrillo, and J. Rosado, A well-posedness theory in measures for some kinetic models of collective motion, Math. Mod. Meth. Appl. Sci., 21(3), 515-539, 2011. 
[4] J.A. Carrillo, M.R. D’Orsogna, and V. Panferov, Double milling in self-propelled swarms from kinetic theory, Kin. Rel. Models, 2, 363-378, 2009.

[5] J.A. Carrillo, M. Fornasier, J. Rosado, and G. Toscani, Asymptotic flocking dynamics for the kinetic Cucker-Smale model, SIAM J. Math. Anal., 42, 218-236, 2010.

[6] J.A. Carrillo, A. Klar, S. Martin, and S. Tiwari, Self-propelled interacting particle systems with roosting force, Math. Mod. Meth. Appl. Sci., 20, 1533-1552, 2010.

[7] F. Cucker and J.G. Dong, Avoiding collisions in flocks, IEEE Trans. Automatic Control, 55, 1238-1243, 2010.

[8] F. Cucker and C. Huepe, Flocking with informed agents, Mathematics in Action, 1, 1-25, 2008.

[9] F. Cucker and E. Mordecki, Flocking in noisy environments, J. Math. Pures Appl., 89, 278-296, 2008.

[10] F. Cucker and S. Smale, Emergent behavior in flocks, IEEE Trans. Automat. Control, 52, 852-862, 2007.

[11] P. Degond and T. Yang, Diffusion in a continuum model of self-propelled particles with alignment interaction, Math. Mod. Meth. Appl. Sci., 20, 1459-1490, 2010.

[12] P. Degond and S. Motsch, Macroscopic limit of self-driven particles with orientation interaction, C.R. Math. Acad. Sci. Paris, 345, 555-560, 2007.

[13] P. Degond and S. Motsch, Large-scale dynamics of the Persistent Turing Walker model of fish behavior, J. Stat. Phys., 131, 989-1022, 2008.

[14] P. Degond and S. Motsch, Continuum limit of self-driven particles with orientation interaction, Math. Mod. Meth. Appl. Sci., 18, 1193-1215, 2008.

[15] R. Duan, M. Fornasier, and G. Toscani, A kinetic flocking model with diffusion, Commun. Math. Phys., 300, 95-145, 2010.

[16] M. Fornasier, J. Haskovec, and G. Toscani, Fluid dynamic description of flocking via PovznerBoltzmann equation, Physica D, 240, 21-31, 2011.

[17] S.Y. Ha, T. Ha, and J. Kim, Asymptotic flocking dynamics for the Cucker-Smale mdoel with the Rayleigh friction, J. Physics A: Math. Theor., 43, 315201, 2010.

[18] S.Y. Ha, E. Jeong, J. Kang, and K.K. Kang, Emergence of multi-cluster configurations from attractive and repulsive interactions, submitted.

[19] S.Y. Ha, M.J. Kang, C. Lattanzio, and B. Rubino, A class of interacting particle systems on the infinite cylinder with flocking phenomena, submitted.

[20] S.Y. Ha, K. Lee, and D. Levy, Emergence of time-asymptotic flocking in a stochastic CuckerSmale system, Commun. Math. Sci., 7, 453-469, 2009.

[21] S.Y. Ha, C. Lattanzio, B. Rubino, and M. Slemrod, Flocking and synchronization of particle models, Quart. Appl. Math., 69, 91-103, 2011.

[22] S.Y. Ha and J.G. Liu, A simple proof of Cucker-Smale flocking dynamics and mean field limit, Commun. Math. Sci., 7, 297-325, 2009.

[23] S.Y. Ha and M. Slemrod, Flocking dynamics of a singularly perturbed oscillator chain and the Cucker-Smale system, J. Dyn. Diff. Equat., 22, 325-330, 2010.

[24] S.Y. Ha and E. Tadmor, From particle to kinetic and hydrodynamic description of flocking, Kin. Rel. Models, 1, 415-435, 2008.

[25] J. Kang, S.Y. Ha, E. Jeong, and K.K. Kang, How do cultural classes emerge from assimilation and distinction? An extension of the Cucker-Smale flocking Model, J. Math. Sociology, to appear.

[26] N.E. Leonard, D.A. Paley, F. Lekien, R. Sepulchre, D.M. Fratantoni, and R.E. Davis, Collective motion, sensor networks and ocean sampling, Proc. IEEE, 95, 48-74, 2007.

[27] S. Motsch and E. Tadmor, A new model for self-organized dynamics and its flocking behavior, J. Stat. Phys., accepted.

[28] D.A. Paley, N.E. Leonard, R. Sepulchre, D. Grunbaum, and J.K. Parrish, Oscillator models and collective motion, IEEE Control Systems, 27, 89-105, 2007.

[29] J. Park, H. Kim, and S.Y. Ha, Cucker-Smale flocking with inter-particle bonding forces, IEEE Tran. Automatic Control, 55, 2617-2623, 2010.

[30] L. Perea, P. Elosegui, and G. Gómez, Extension of the Cucker-Smale control law to space flight formation, J. Guidance, Control and Dynamics, 32, 526-536, 2009.

[31] J. Shen, Cucker-Smale flocking under hierarchical leadership, SIAM J. Appl. Math., 68, 694$719,2007$.

[32] J. Toner and Y. Tu, Flocks, herds, and schools: A quantitative theory of flocking, Physical Review E., 58, 4828-4858, 1998.

[33] C.M. Topaz and A.L. Bertozzi, Swarming patterns in a two-dimensional kinematic model for biological groups, SIAM J. Appl. Math., 65, 152-174, 2004.

[34] T. Vicsek, A. Czirók, E. Ben-Jacob, I. Cohen, and O. Schochet, Novel type of phase transition in a system of self-driven particles, Phys. Rev. Lett., 75, 1226-1229, 1995. 